\title{
MODELAGEM DE UM CONDICIONADOR DE AR DE ALTA PRECISÃO PARA USO EM PROCESSAMENTO AGRÍCOLA
}

\author{
MAURI FORTES ${ }^{1}$, DÉLCIO A. FERREIRA ${ }^{2}$, WANYR R. FERREIRA ${ }^{3}$, \\ ANTÔNIO C. DE SOUZA ${ }^{4}$
}

\begin{abstract}
RESUMO: Apresenta-se neste trabalho, estudo detalhado sobre a modelagem de um condicionador de ar com controle acurado de temperatura e umidade relativa. Desenvolveu-se um programa de computador que permite predizer o comportamento do sistema sob diferentes condições psicrométricas e de vazão do ar de entrada. O modelo global físico-matemático inclui equações de balanço de massa e de energia para três diferentes volumes de controle que compõem o condicionador. Modelaram-se os processos de mistura, evaporação (ou condensação) simultânea de uma superfície d'água e de uma gota e o aquecimento de corrente de ar, separadamente. Propõem-se expressões semi-empíricas simples para os coeficientes de transferência de calor e massa inerentes ao processo de evaporação sobre uma superfície d'água e um modelo simplificado para o sistema de spray. Construiu-se um condicionador de ar no qual foram feitos testes experimentais para o ajuste das constantes que aparecem nas expressões para os coeficientes de transferência. Os dados obtidos validaram o modelo global, com precisão aceitável para projetos de engenharia.
\end{abstract}

PALAVRAS-CHAVE: ambiente controlado, condicionamento de ar, câmara climática.

\section{MODELING OF A HIGH PRECISION AIR CONDITIONER FOR USE IN AGRICULTURAL PROCESSING}

\begin{abstract}
In this work, a detailed procedure for the analysis of an accurately controlled air conditioner is presented. A computer program, that allows predicting the behavior of the system under different psychrometric conditions and different input air mass flow rates, was developed. The global physical-mathematical model includes mass and energy conservation equations for three different control volumes that compose the conditioner. Thus, the processes of mixture of air, simultaneous evaporation (or condensation) from a water surface and from a drop, and the airstream heating are modeled separately. Simple semi-empiric expressions for heat and mass coefficients inherent to the evaporation process on a water surface are proposed as well a simplified model for the spray system. Experimental tests made on an air-conditioner allowed to obtain the constants that appear in the expressions for the heat and mass transfer coefficients and to validate the global model, within an engineering acceptable precision level.
\end{abstract}

KEYWORDS: controlled ambient, air conditioning, climatic chamber.

\section{INTRODUÇÃO}

O controle de temperatura e de umidade relativa do ar é importante em várias áreas de desenvolvimento tecnológico. No setor agrícola há necessidade de ambientes controlados em estudos experimentais de propriedades físicas e de movimentação de umidade em solos, plantas e produtos agrícolas. A criação de climas artificiais, em estufas, requer controle rigoroso de temperatura e umidade relativa, como no caso de fertilização in vitro (FIRST \& PARRISH, 1999). Não existem, na literatura pesquisada, estudos detalhados sobre modelagem de equipamentos reais

\footnotetext{
${ }^{1} \mathrm{PhD}$, Prof. Titular, Centro Universitário UNA, R. Aimorés, 1451, Belo Horizonte - MG, mauri.fortes@ terra.com.br.

${ }^{2}$ Eng ${ }^{\mathrm{o}}$ Mecânico, Mestre, Departamento de Engenharia Mecânica, UFMG, Belo Horizonte - MG.

${ }^{3}$ Docteur, Profa. Adjunta, Centro Universitário UNA, Belo Horizonte - MG.

${ }^{4}$ Especialista em Energia Térmica, Consciente Ltda., Belo Horizonte - MG.

Recebido pelo Conselho Editorial em: 21-9-2004

Aprovado pelo Conselho Editorial em: 9-6-2006
} 
de controle preciso das condições termodinâmicas do ar, embora existam equipamentos comerciais, tais como o Aminco-Aire (Aminco).

Para o dimensionamento de equipamentos de laboratório de condicionamento de ar ou de controle mais acurado de temperatura e umidade relativa, é fundamental conhecer os processos de transferência de calor e massa em superfícies molhadas e em gotas atomizadas (spray). A transferência de calor e massa entre uma superfície d'água e o ar ambiente ocorre com mudança de fase, ou seja, condensação de vapor d'água do fluxo de ar ou evaporação na superfície molhada. CHILTON \& COLBURN, citados por BIRD et al. (2001), desenvolveram pela primeira vez, em 1934, uma analogia entre a transferência de calor e a transferência de massa. Eles admitiram a equivalência entre dois grupos adimensionais, associados às transferências de calor e massa. As expressões associadas levam a valores calculados coerentes com os experimentais associados à camada limite (laminar ou turbulenta) sobre placas planas, a baixas taxas de transferência na superfície (BIRD et al., 2001).

Outros estudos de transporte simultâneo de calor e massa a partir de uma superfície livre contendo líquido ou de uma placa plana porosa úmida também foram realizados, quer de forma teórica, quer de forma experimental (KEEY, 1972; FERREIRA et al., 1993a e 1993b). Expressões e dados empíricos para taxas de evaporação e condensação de gotas de ar ou vapor superaquecido encontram-se em YULE et al. (2002), NASR et al. (2002), SINGH \& HELDMAN (2001) e BONACINA et al. (1979). O dimensionamento do processo de troca térmica entre gotas e ar definirá o nível de saturação do ar à saída da câmara condicionadora. O objetivo básico do projeto de uma câmara condicionadora é fornecer as condições geométricas de transferência de calor e massa e termodinâmicas de forma a assegurar que o ar, que entra na câmara, possa ser condicionado a uma temperatura de ponto de orvalho desejado e, após aquecimento, atingir a temperatura de bulbo seco desejada.

Em equipamentos condicionadores de ar, os processos de transferência de calor e massa ocorrem dentro de estruturas complexas e sob condições de escoamento que não se ajustam adequadamente aos modelos da literatura, fato que impede soluções numéricas ou analíticas. Uma análise, por técnica numérica envolvendo elementos finitos ou volumes finitos, seria bastante complexa do ponto de vista computacional.

Este trabalho teve como objetivos apresentar a modelagem físico-matemática, empíricoteórica, de um condicionador de ar de alta precisão, e desenvolver expressões adimensionais para projeto; a modelagem deverá ser validada por meio de testes experimentais em um condicionador real de ar de alta precisão, construído especificamente para este estudo, a partir dos dados de construção e funcionamento de um condicionador de ar comercial.

\section{MATERIAL E MÉTODOS}

\section{Descrição do equipamento de condicionamento de ar e instrumentos de controle e medição}

Tendo por base um condicionador de ar de laboratório comercial, construiu-se outro similar, para efeito de avaliação experimental. Assim, mantendo-se as características geométricas, Figuras 1 (esquema) e 2 (foto), adicionaram-se novos componentes elétricos e eletrônicos, ou seja, controladores, resistores de potência variável, ventilador, sistemas de controle elétrico, sondas do tipo Pt-100 e sistema de refrigeração de potência aproximada a do condicionador de referência.

Testou-se e modelou-se o condicionador climático modificado, composto de dois compartimentos principais, sendo o compartimento superior visto na Figura 2. O compartimento inferior do condicionador consistiu de uma unidade de refrigeração e uma bomba d'água. O compartimento superior contém os equipamentos usados no condicionamento de ar e divide-se em três câmaras: câmara de entrada do fluxo de ar ambiente $(15 \mathrm{~cm} \times 75 \mathrm{~cm})$, com reservatório de água; câmara do spray $(45 \mathrm{~cm} \times 75 \mathrm{~cm})$, com reservatório de água, cinco bocais de spray e um eliminador de gotas, e câmara de saída do fluxo de ar $(60 \mathrm{~cm}$ x $25 \mathrm{~cm})$, que contém um exaustor e um aquecedor elétrico de ar (Figura 1). A câmara de entrada é separada da câmara do spray por 
uma parede (Figura 2), com uma abertura que permite a conexão entre as câmaras; nelas foram colocados o evaporador do sistema de resfriamento e um resistor de forma a se poder aquecer ou resfriar a água do reservatório e do spray. A água usada no spray provém da camada de água (reservatório) das câmaras de entrada e de spray.

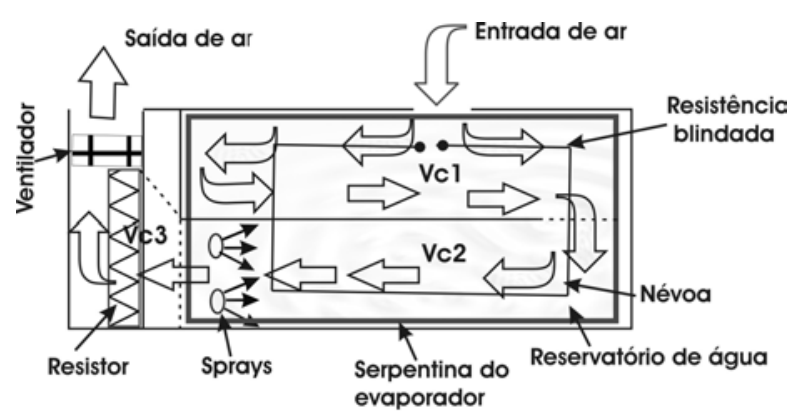

FIGURA 1. Câmara condicionadora de ar.

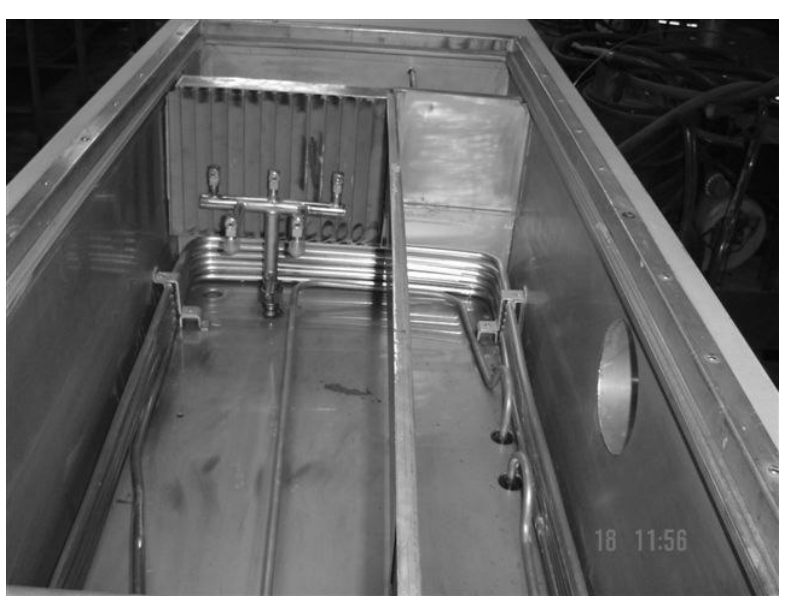

FIGURA 2. Foto da câmara condicionadora de ar.

O sistema de refrigeração compôs-se de um compressor Tecumseh-RG181RS, de potência nominal de refrigeração de $2,9 \mathrm{~kW}$, às temperaturas de evaporação e condensação iguais a $7,2{ }^{\circ} \mathrm{C} \mathrm{e}$ $54,4{ }^{\circ} \mathrm{C}$, respectivamente. As potências elétricas do resistor blindado (dentro da água) e do resistor de aquecimento do ar à saída eram de $4,5 \mathrm{~kW}$ e $3 \mathrm{~kW}$, respectivamente. O ventilador de aspiração tinha potência de 1/8 HP. Controladores - indicadores tipo PID NOVUS NR-1100, com precisão de $0,1{ }^{\circ} \mathrm{C}$, atuando sobre os sistemas de refrigeração e de aquecimento, controlavam a temperatura da água da câmara, da água que saía pelo spray, mantendo-a próxima à temperatura de orvalho desejada e, também, a temperatura de bulbo seco do ar, à saída da câmara. A umidade relativa também era medida por um controlador indicador NOVUS NR-1100, com precisão de $0,1 \%$, e por um sensor AHC-80, desenvolvido pela Full-Gauge, com precisão de 1\%. A bomba d'água utilizada nos sprays tinha potência de $1 \mathrm{HP}$; com essa bomba e com o orifício de spray selecionado, o diâmetro médio das gotas, conforme especificação do fabricante (Modelo 29JAUCO automatic air atomizing spray nozzle - Spraying Systems), era de 1,6 mm. A movimentação de água no reservatório, causada pela sucção da bomba, aumenta a transferência convectiva de calor entre a água e a serpentina e o resistor blindado; por esse motivo, não se colocaram evaporadores em formato de múltiplas voltas (serpentinas) ou resistores aletados; a câmara comercial, base para o protótipo deste trabalho, também não possui instrumentos de aumento de troca convectiva de calor.

\section{Equações de conservação para os volumes de controle}

Para efeito de modelagem, dividiu-se a câmara condicionadora em três volumes de controle (Vc1, Vc2 e Vc3), conforme Figura 1. As equações usadas para modelar termodinamicamente o equipamento em cada volume de controle encontram-se na Tabela 1.

Nas equações da Tabela 1, o índice 1 refere-se a valores de entrada no Vc1; o índice 2, a valores de saída do Vc1 que entram no Vc2; o índice 3, a valores de saída do Vc2 que entram no Vc3, e o índice 4, a valores à saída do condicionador. Ainda na Tabela 1, sp refere-se a spray; ev, à água evaporada da superfície; $\dot{\mathrm{m}}=$ vazão mássica de ar úmido $\left(\mathrm{kg} \mathrm{s}^{-1}\right) ; \dot{\mathrm{m}}_{\mathrm{a}}=$ vazão mássica de ar $\operatorname{seco}\left(\mathrm{kg} \mathrm{s}^{-1}\right) ; \mathrm{h}=$ entalpia específica $\left(\mathrm{kJ} \mathrm{kg}^{-1}\right) ; \mathrm{T}=$ temperatura $(\mathrm{K}) ; \mathrm{w}=$ umidade absoluta $\left(\mathrm{kg} \mathrm{kg}^{-1}\right)$;

$\dot{q}_{s p}=$ taxa de entrada da entalpia da água do spray $(\mathrm{W}) ; \dot{q}_{r}=$ potência elétrica do resistor $(\mathrm{W})$, e $\mathrm{c}_{\mathrm{pa}}$ = calor específico do ar $\left(\mathrm{kJ} \mathrm{kg}^{-1} \mathrm{~K}^{-1}\right)$. 
TABELA 1. Equações de conservação para os volumes de controle.

\begin{tabular}{lll}
\hline & \multicolumn{1}{c}{ Massa } & \multicolumn{1}{c}{ Energia } \\
\hline Vc1 & $\dot{\mathrm{m}}_{2}-\dot{\mathrm{m}}_{1}-\dot{\mathrm{m}}_{\mathrm{ev}, 1}=0$ & $\dot{\mathrm{m}}_{1} \mathrm{~h}_{1}+\dot{\mathrm{q}}_{\mathrm{r}, 1}+\dot{\mathrm{m}}_{\mathrm{ev}, 1} \mathrm{~h}_{\mathrm{ev}, 1}-\dot{\mathrm{m}}_{2} \mathrm{~h}_{2}=0$ \\
& $\dot{\mathrm{m}}_{\mathrm{a}}-\dot{\mathrm{m}}_{1}\left(1-\mathrm{w}_{1}\right)=0$ & $\mathrm{~T}_{2}-\left[\left(1+\mathrm{w}_{2}\right) \mathrm{h}_{2}-\mathrm{w}_{2} \mathrm{~h}_{\mathrm{v} 2}\right] / \mathrm{c}_{\mathrm{pa}}=0$ \\
& $\mathrm{w}_{2}-\dot{\mathrm{m}}_{2} / \dot{\mathrm{m}}_{\mathrm{a}}+1=0$ & \\
\hline \multirow{2}{*}{$\mathrm{Vc} 2$} & $\dot{\mathrm{m}}_{3}-\dot{\mathrm{m}}_{2}-\dot{\mathrm{m}}_{\mathrm{ev}, 2}-\dot{\mathrm{m}}_{\mathrm{sp}}=0$ & $\dot{\mathrm{m}}_{2} \mathrm{~h}_{2}+\dot{\mathrm{m}}_{\mathrm{ev}, 2} \mathrm{~h}_{\mathrm{ev}, 2}-\dot{\mathrm{m}}_{3} \mathrm{~h}_{3}+\dot{\mathrm{q}}_{\mathrm{sp}}=0$ \\
& $\mathrm{w}_{3}-\dot{\mathrm{m}}_{3} / \dot{\mathrm{m}}_{\mathrm{a}}+1=0$ & $\mathrm{~T}_{3}-\left[\left(1+\mathrm{w}_{3}\right) \mathrm{h}_{3}-\mathrm{w}_{3} \mathrm{~h}_{\mathrm{v} 3}\right] / \mathrm{c}_{\mathrm{pa}}=0$ \\
\hline \multirow{2}{*}{$\mathrm{Vc} 3$} & $\dot{\mathrm{m}}_{4}-\dot{\mathrm{m}}_{3}=0$ & $\dot{\mathrm{m}}_{3} \mathrm{~h}_{3}+\dot{\mathrm{q}}_{\mathrm{r}, 3}-\dot{\mathrm{m}}_{4} \mathrm{~h}_{4}=0$ \\
& & $\mathrm{~T}_{4}-\left[\left(1+\mathrm{w}_{4}\right) \mathrm{h}_{4}-\mathrm{w}_{4} \mathrm{~h}_{\mathrm{v} 4}\right] / \mathrm{c}_{\mathrm{pa}}=0$
\end{tabular}

Nota: Utilizou-se o sistema SI em todo este trabalho.

As unidades das outras variáveis usadas na modelagem e simulação estão especificadas na Tabela 2, que apresenta as equações de regressão obtidas, quer neste trabalho, quer da literatura, para as propriedades termodinâmicas da água e do ar úmido, usadas na simulação.

TABELA 2. Equações de propriedades termodinâmicas e térmicas para o ar úmido (T é temperatura em $\mathrm{K} ; \mathrm{T}_{\mathrm{c}}, \mathrm{em}^{\circ} \mathrm{C}$ )

\begin{tabular}{|c|c|c|c|}
\hline Propriedade & Equação & $\begin{array}{l}\text { Validade } \\
\text { Erro }(\%) \\
\end{array}$ & Ref. \\
\hline Pressão de vapor d'água saturado $(\mathrm{kPa})$ & $\mathrm{Ps}=\exp (25,53 / \mathrm{T}) / 1000$ & $\begin{array}{c}7<\mathrm{T}_{\mathrm{c}}<80^{\circ} \mathrm{C} \\
0,03 \%\end{array}$ & $* *$ \\
\hline Umidade absoluta $\left(\mathrm{kg} \mathrm{kg}^{-1}\right)$ & $\begin{array}{l}\mathrm{W}=0,62192 \mathrm{P}_{\mathrm{S}} \phi /\left(\mathrm{P}-\mathrm{P}_{\mathrm{S}} \phi\right), \text { em que } \mathrm{P}= \\
\text { pressão total e } \phi=\text { umidade relativa do ar }\end{array}$ & - & 1 \\
\hline Constante do ar úmido, como gás perfeito & $\begin{array}{l}\mathrm{R}_{\mathrm{a}}=\left[\mathrm{R}_{\mathrm{u}} /(1+\mathrm{w})\right](1 / 28,97+\mathrm{w} / 18,16) \mathrm{em} \\
\text { que, } \mathrm{R}_{\mathrm{u}}=\text { constante universal dos gases }\end{array}$ & - & 3 \\
\hline Calor específico do ar seco $\left(\mathrm{kJ} \mathrm{kg}^{-1} \mathrm{~K}^{-1}\right)$ & $\mathrm{c}_{\mathrm{ps}}=1,003+9,89110^{-5} \mathrm{~T}$ & $\begin{array}{c}5<\mathrm{T}_{\mathrm{c}}<80^{\circ} \mathrm{C} \\
0,3 \%\end{array}$ & $* *$ \\
\hline Calor específico do ar úmido $\left(\mathrm{kJ} \mathrm{kg}^{-1} \mathrm{~K}^{-1}\right)$ & $\begin{array}{l}\mathrm{C}_{\mathrm{pa}}=\mathrm{c}_{\mathrm{ps}}+\mathrm{w}_{\mathrm{pv}} \text {, em que, } \mathrm{c}_{\mathrm{pv}} \text { é o calor } \\
\text { específico do vapor d'água }\end{array}$ & - & 2 \\
\hline Viscosidade dinâmica do ar úmido ( $\mathrm{kPa}$ s) & $\mu=4,6710^{-8} \mathrm{~T}+4,5310^{-6}$ & $\begin{array}{c}4<\mathrm{T}_{\mathrm{c}}<80^{\circ} \mathrm{C} \\
0,4 \%\end{array}$ & $* *$ \\
\hline Vazão mássica de ar seco $\left(\mathrm{kg} \mathrm{s}^{-1}\right)$ & $\begin{array}{l}\dot{\mathrm{m}}_{\mathrm{as}}=\dot{\mathrm{m}}_{\mathrm{a}} /(1+\mathrm{w}), \text { em que } \dot{\mathrm{m}}_{\mathrm{a}}=\text { vazão } \\
\text { mássica de ar úmido }\end{array}$ & - & 1 \\
\hline Entalpia do vapor d'água $\left(\mathrm{kJ} \mathrm{kg}^{-1}\right)$ & $\mathrm{H}_{\mathrm{v}}=2501,84+1,81334(\mathrm{~T}-273,15)$ & $\begin{array}{c}4<\mathrm{T}_{\mathrm{c}}<80^{\circ} \mathrm{C} \\
0,6 \%\end{array}$ & ** \\
\hline Entalpia da água líquida $\left(\mathrm{kJ} \mathrm{kg}^{-1}\right)$ & $\mathrm{H}_{\mathrm{L}}=0,1942+4,181(\mathrm{~T}-273,15)$ & $\begin{array}{c}4<\mathrm{T}_{\mathrm{c}}<80^{\circ} \mathrm{C} \\
0,57 \%\end{array}$ & $* *$ \\
\hline Entalpia do ar úmido $\left(\mathrm{kJ} \mathrm{kg}^{-1}\right)$ & $\mathrm{H}_{\mathrm{a}}=\mathrm{c}_{\mathrm{ps}}(\mathrm{T}-273,15)+\mathrm{w} \mathrm{H}_{\mathrm{v}}$ & - & 3 \\
\hline Condutividade térmica do ar úmido $\left(\mathrm{W} \mathrm{m}^{-1} \mathrm{~K}^{-1}\right)$ & $\mathrm{K}_{\mathrm{a}}=\left(7,33110^{-5} \mathrm{~T}+0,004071\right) / 1000$ & $\begin{array}{c}4<\mathrm{T}_{\mathrm{c}}<80^{\circ} \mathrm{C} \\
0,4 \%\end{array}$ & $* *$ \\
\hline Condutividade térmica da água $\left(\mathrm{W} \mathrm{m}^{-1} \mathrm{~K}^{-1}\right)$ & $\mathrm{K}_{\mathrm{L}}=0,5630+0,001530(\mathrm{~T}-273,15)$ & $\begin{array}{c}4<\mathrm{T}_{\mathrm{c}}<60^{\circ} \mathrm{C} \\
0,87 \%\end{array}$ & $* *$ \\
\hline Difusividade de massa na superfície molhada $\left(\mathrm{m}^{2} \mathrm{~s}^{-1}\right)$ & $\begin{array}{l}\mathrm{D}_{1}=\left(22,29+0,07435 \mathrm{~T}+0,0033203 \mathrm{~T}^{0,2}-\right. \\
\left.4,02110^{-5} \mathrm{~T}^{0,3}+2,63410^{-7} \mathrm{~T}^{0,4}\right) 10^{-6}\end{array}$ & $\begin{array}{c}4<\mathrm{T}_{\mathrm{c}}<80^{\circ} \mathrm{C} \\
0,67 \%\end{array}$ & $* *$ \\
\hline Difusividade de massa da água no spray $\left(\mathrm{m}^{2} \mathrm{~s}^{-1}\right)$ & $\mathrm{D}_{2}=\left(4,5310^{-7} \mathrm{~T}^{2,334}\right) 10^{-4}$ & - & 4 \\
\hline $\begin{array}{l}\text { Coeficiente convectivo entre a gota d'água e o ar } \\
\text { úmido }\left(\mathrm{kW} \mathrm{m} \mathrm{K}^{-1}\right)\end{array}$ & $h_{q}=\left[2+0,6\left(\phi_{g} v_{g} \rho_{a} / \mu\right)^{1 / 2}\left(C p \mu / k_{a}\right)^{1 / 3}\right]\left(k_{L} / \phi\right)$ & - & 3,4 \\
\hline $\begin{array}{l}\text { Coeficiente de transferência de massa entre a gota e o } \\
\text { ar úmido }\left(\mathrm{kg} \mathrm{m}^{-2} \mathrm{~s}^{-1}\right)\end{array}$ & $h_{m}=\left[2+0,6\left(\phi_{g} v_{g} \rho / \mu\right)^{1 / 2}\left(\mu / \rho D_{2}\right)^{1 / 3}\right]\left(k_{1} D_{2} / \phi\right)$ & - & 3,4 \\
\hline
\end{tabular}


Transporte de calor e massa entre o ar e a superfície molhada, no interior da câmara climática

Os números adimensionais usados neste trabalho encontram-se na Tabela 3.

TABELA 3. Números ou grupos adimensionais usados neste trabalho.

\begin{tabular}{lclr}
\hline Stanton, $\mathrm{St}$ & $\mathrm{h}_{\mathrm{q}} /\left(\rho \mathrm{c}_{\mathrm{p}} \mathrm{U}\right)$ & Stanton, para transferência de massa, $\mathrm{St}_{\mathrm{m}}$ & $\mathrm{h}_{\mathrm{m}} / \mathrm{U}$ \\
\hline Reynolds, Re & $\rho \mathrm{UL} / \mu$ & Biot, Bi & $\mathrm{h}_{\mathrm{q}} \mathrm{d} / \mathrm{k}_{\mathrm{L}}$ \\
\hline Prandtl, Pr & $\mu \mathrm{c}_{\mathrm{p}} / \mathrm{k}_{\mathrm{a}}$ & Schmidt, Sc & $\mu /(\rho \mathrm{D})$ \\
\hline
\end{tabular}

$\mathrm{Na}$ Tabela 3, $\mathrm{h}_{\mathrm{q}}$ e $\mathrm{h}_{\mathrm{m}}$ são, respectivamente, os coeficientes de transferência de calor $\left(\mathrm{kW} \mathrm{m}^{-2} \mathrm{~K}^{-1}\right)$ e massa $\left(\mathrm{kg} \mathrm{m}^{-2} \mathrm{~s}^{-1}\right) ; \mathrm{k}_{\mathrm{a}}$ e $\mathrm{k}_{\mathrm{L}}$ referem-se, respectivamente, às condutividades térmicas do ar úmido e da água $\left(\mathrm{W} \mathrm{m}^{-1} \mathrm{~K}^{-1}\right) ; \rho, \mu, \mathrm{c}_{\mathrm{p}} \mathrm{e} \mathrm{D}$ referem-se, respectivamente, à massa específica $\left(\mathrm{kg} \mathrm{m}^{-3}\right)$, viscosidade dinâmica $(\mathrm{kPa} \mathrm{s})$, calor específico $\left(\mathrm{kJ} \mathrm{kg}^{-1} \mathrm{~K}^{-1}\right)$ e coeficiente de difusão entre $\mathrm{o}$ ar úmido e a água $\left(\mathrm{m}^{2} \mathrm{~s}^{-1}\right)$; d é o diâmetro da gota $(\mathrm{m})$; L é uma dimensão característica (largura da passagem do ar ou diâmetro da gota, $\mathrm{m})$, e U é a velocidade média do ar ( $\left.\mathrm{m} \mathrm{s}^{-1}\right)$.

A velocidade média (U), sobre ou acima da superfície plana de água ou em cada seção ou volume de controle, foi estimada a partir da medição da vazão na seção circular de entrada da unidade condicionadora. Assim, media-se a velocidade do ar, no duto circular de entrada (100 mm de diâmetro), em diferentes pontos, ao longo de diâmetros perpendiculares entre si, por meio de anemômetro eletrônico tipo ventoinha (Modelo ExTech 45118), com precisão de $0,1 \mathrm{~m} \mathrm{~s}^{-1}$, na faixa investigada de temperaturas. A partir da velocidade média no duto de entrada, calculava-se a vazão de ar na câmara condicionadora, por integração numérica. Dividindo-se a vazão pela área da seção transversal de interesse, obtinha-se o valor médio da velocidade (U), nessa seção ou volume de controle. Correções de massa específica para diferentes condições psicrométricas eram feitas por meio de balanços clássicos de energia e massa (KEEY, 1972).

Os coeficientes de transferência de calor e massa, com mudança de fase, entre a superfície molhada e o ar úmido, foram avaliados por meio da analogia de Chilton-Colburn (BIRD et al., 2001). Supôs-se, portanto, a equivalência entre dois grupos adimensionais, $\mathrm{J}_{\mathrm{e}} \mathrm{J}_{\mathrm{m}}$, definidos por:

$$
\mathrm{J}=\mathrm{StPr}^{2 / 3}=\mathrm{J}_{\mathrm{m}}=\mathrm{St}_{\mathrm{m}} \mathrm{Sc}^{2 / 3}=\mathrm{A}_{1} \operatorname{Re}^{\mathrm{B}_{1}}
$$

em que,

$\mathrm{A}_{1}, \mathrm{~B}_{1}$ - constantes empíricas a serem determinadas.

Neste trabalho, o ajuste do modelo teórico aos dados experimentais implicou determinação dessas constantes para a situação física e geométrica da câmara climática. Pode-se desenvolver uma expressão para avaliar o coeficiente de transferência de massa, $\mathrm{h}_{\mathrm{m}}$, a partir da definição do número de Stanton local (Tabela 3) e da eq.(1):

$$
\mathrm{h}_{\mathrm{m}}=\mathrm{A}_{1} \operatorname{Re}^{\mathrm{B}_{1}} \mathrm{USc}^{-2 / 3}
$$

em que,

$\mathrm{U}$ - velocidade média do ar úmido sobre a superfície, $\mathrm{m} \mathrm{s}^{-1}$.

A taxa de condensação ou evaporação de água $(\mathrm{dN})$, no elemento de área $(\mathrm{dA})$ da superfície molhada, é dada por TREYBAL (1981):

$$
\mathrm{dN}=\mathrm{dA} \rho \mathrm{h}_{\mathrm{m}} \ln \left(\mathrm{P}_{\mathrm{B} 2} / \mathrm{P}_{\mathrm{B} 1}\right)
$$

em que,

$\rho$ - massa específica do ar úmido, $\mathrm{kg} \mathrm{m}^{-3}$, e

$\mathrm{P}_{\mathrm{B} 1}, \mathrm{P}_{\mathrm{B} 2}$ - pressão parcial do vapor d'água no ar, junto à superfície e na corrente livre, respectivamente, $\mathrm{N} \mathrm{m}^{-2}$. 
Essa equação permite determinar a taxa de água condensada sobre a superfície molhada (processo de desumidificação do ar), quando o valor de $\ln \left(\mathrm{P}_{\mathrm{B} 1} / \mathrm{P}_{\mathrm{B} 2}\right)$ for negativo, e a vazão mássica de água evaporada da superfície (umidificação do ar), quando o valor de $\ln \left(\mathrm{P}_{\mathrm{B} 1} / \mathrm{P}_{\mathrm{B} 2}\right)$ for positivo.

Neste trabalho, subdividiu-se a área da superfície para a transferência de massa em elementos da área $\mathrm{dA}$, pois à medida que o fluxo de ar avança sobre a superfície molhada, o seu estado é variável. A eq.(3) foi, então, integrada numericamente e aproximadamente para toda a superfície real, supondo escoamento linear sobre as superfícies, levando à taxa total de água evaporada ou condensada $(\mathrm{N})$.

Analogamente, o coeficiente de transferência de calor entre a superfície molhada e o ar úmido, escoando paralelamente a essa superfície, em presença de transferência de massa, também foi obtido utilizando a analogia de Chilton-Colburn (BIRD et al., 2001); assim, da definição de St, na Tabela 3 e da eq. (1) conclui-se que:

$$
\mathrm{h}_{\mathrm{q}}=\mathrm{A}_{3} \operatorname{Re}^{\mathrm{B}} \operatorname{Pr}^{-2 / 3} \rho \mathrm{c}_{\mathrm{p}} \mathrm{U}
$$

em que,

$\mathrm{A}_{3}$ - constante empírica, a ser determinada.

A taxa de calor total (dq) transferida entre o elemento de área (dA) da superfície molhada e o fluxo de ar úmido paralelo a essa superfície, na presença de transferência de massa, é:

$$
\mathrm{dq}_{\mathrm{t}}=\mathrm{dq}_{\mathrm{s}}+\mathrm{H}_{\mathrm{lv}} \mathrm{dN}
$$

em que,

$\mathrm{H}_{\mathrm{lv}}$ - entalpia latente específica da água, $\mathrm{kJ} \mathrm{kg}^{-1}$, e

$\mathrm{dq}_{\mathrm{s}}$ - taxa de calor sensível, W.

Em termos da teoria de filme, a seguinte expressão fornece a taxa de calor sensível oriundo do movimento de massa (evaporação ou condensação), dN, sob a superfície dA (TREYBAL, 1981):

$$
\mathrm{dq}_{\mathrm{s}}=\frac{\mathrm{dAdNc}_{\mathrm{p}}\left(\mathrm{T}_{3}-\mathrm{T}_{2}\right)}{1-\mathrm{e}^{-\mathrm{dNc_{ \textrm {p } } / \mathrm { h }}}}
$$

A eq.(5) foi integrada numericamente para a área total da superfície da água.

\section{Transporte de calor e massa entre gotas atomizadas e ar úmido}

Durante a trajetória da gota no ar, a sua temperatura, diâmetro e coeficientes de transferência de calor e massa variam. Supondo que a resistência interna à troca de calor no corpo da gota seja desprezível em relação à resistência entre sua superfície e o meio, isto é, supondo que Biot $<0,1$, a variação da sua temperatura ao longo da trajetória pode ser estimada pela expressão (HOLMAN, 2002):

$$
-\mathrm{m}_{\mathrm{G}} \mathrm{c}_{\mathrm{p}} \frac{\mathrm{dT}_{\mathrm{G}}}{\mathrm{dt}}=\mathrm{hA}_{\mathrm{G}}\left(\mathrm{T}_{\mathrm{G}}-\mathrm{T}_{\mathrm{ar}}\right)+\dot{\mathrm{m}}_{\mathrm{ev}} \mathrm{H}_{\mathrm{lv}}
$$

em que,

$\mathrm{m}_{\mathrm{G}}$ - massa de uma única gota, $\mathrm{kg}$;

$\mathrm{T}_{\mathrm{G}}$ - temperatura de uma única gota, $\mathrm{K}$, e

$\mathrm{A}_{\mathrm{G}}$ - área superficial de uma única gota, $\mathrm{m}^{2}$.

Essa equação diferencial tem como solução:

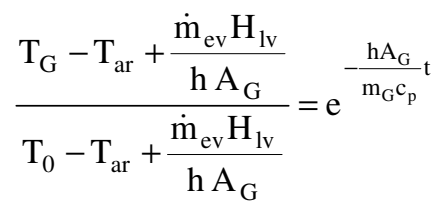


em que,

$\mathrm{T}_{0}$ - temperatura inicial da gota, $\mathrm{K}$.

A taxa de água evaporada, $\dot{\mathrm{m}}_{\mathrm{ev}}\left(\mathrm{kg} \mathrm{s}^{-1}\right)$, de uma única gota atomizada, pode ser avaliada pela expressão (BIRD et al., 2001):

$$
\dot{\mathrm{m}}_{\mathrm{ev}}=\mathrm{h}_{\mathrm{gm}} \pi \mathrm{d}^{2} \frac{\left(\mathrm{x}_{0}-\mathrm{x}_{\infty}\right)}{\left(1-\mathrm{x}_{0}\right)}
$$

em que,

d - diâmetro de uma gota d'água, m;

$\mathrm{x}_{0}, \mathrm{x}_{\infty}$ - fração molar de vapor no ar, junto à superfície e na corrente livre, respectivamente, e $\mathrm{h}_{\mathrm{gm}}$ - coeficiente de transferência de massa entre uma gota d'água e o ar, $\mathrm{kg} \mathrm{m}^{-2} \mathrm{~s}^{-1}$.

Os coeficientes empíricos de transferência de calor e de massa podem ser, respectivamente, obtidos das expressões empíricas de BIRD et al. (2001) e FIRST \& PARRISH (1987):

$$
h_{g}=\left(2,0+0,6 \operatorname{Re}^{1 / 2} \operatorname{Pr}^{1 / 3}\right) \frac{k_{a}}{d} \quad \text { e } \quad h_{g m}=\left(2,0+0,6 \operatorname{Re}^{1 / 2} \operatorname{Sc}^{1 / 3}\right) \frac{\left(w \rho_{a r} D\right)}{d}
$$

A energia total trocada entre o ar e as gotas, durante todo o seu percurso, pode ser obtida por meio da expressão:

$$
\mathrm{q}_{\mathrm{sp}}=-\mathrm{n}_{\mathrm{g}} \int_{\mathrm{t}_{1}}^{\mathrm{t}_{\mathrm{f}}}\left\{\mathrm{h}_{\mathrm{g}} \mathrm{A}_{\mathrm{g}}\left(\mathrm{T}_{\mathrm{g}}-\mathrm{T}\right)+\dot{\mathrm{m}}_{\mathrm{ev}} \mathrm{h}_{\mathrm{v}}\right\} \mathrm{dt}
$$

em que,

$\mathrm{n}_{\mathrm{g}}$ - número de gotas atomizadas pelo spray, na unidade de tempo.

O número de gotas na unidade de tempo pode ser estimado pela expressão:

$$
\mathrm{n}_{\mathrm{g}}=\dot{\mathrm{m}}_{\mathrm{b}} /\left(\rho_{\mathrm{L}} \mathrm{V}\right)
$$

em que,

$\dot{\mathrm{m}}_{\mathrm{b}}$ - vazão de água bombeada, $\mathrm{kg} \mathrm{s}^{-1}$, e

V - volume da gota suposta esférica, à saída do spray, considerando que as gotas constituam uma massa única, $\mathrm{m}^{3}$.

\section{Metodologia de solução do sistema de equações não-lineares}

As equações dos balanços de energia e massa (Tabela 1) foram resolvidas para o Vc1, e seus resultados, utilizados na solução das equações do Vc2 e, analogamente, obtiveram-se as soluções associadas ao Vc3. Os dados de entrada são: temperatura de bulbo seco na entrada da câmara $\left(\mathrm{T}_{1}\right)$, temperaturas da água da superfície molhada $\left(\mathrm{T}_{\text {sup }}\right)$ e do spray $\left(\mathrm{T}_{\mathrm{sp}}\right)$, umidade relativa do ar na entrada da câmara $\left(\phi_{1}\right)$, velocidade do ar na entrada da câmara $\left(\mathrm{U}_{1}\right)$ e pressão barométrica local $(\mathrm{P})$.

Os balanços de energia e massa referentes aos volumes de controle 1 e 2 requerem o cálculo das taxas de transferência de calor e massa do spray (gotas) e da superfície molhada. Para isso, cada volume de controle foi subdividido em vários subvolumes de controle, e cada conjunto de equações foi resolvido pelo método de Newton-Raphson, em cada subvolume. Dessa forma, o balanço global de energia e massa foi realizado por meio de integração numérica, sendo consideradas 100 subdivisões em cada volume de controle. A programação foi efetuada por meio do "software" Power FORTRAN da Microsoft, usando o pacote IMSL FORTRAN NUMERICAL LIBRARY, interno a ele, e os seus diversos algoritmos numéricos, com controle de erro, usandose, também, o MATHEMATICA. Os dados obtidos por ambos os "softwares" coincidiram no limite do erro de truncamento (16 casas decimais). O tempo médio de simulação foi de três minutos; todo o programa em FORTRAN ou MATHEMATICA não ocupava mais de $50 \mathrm{kB}$. A integração da eq.(11) foi efetuada levando-se em conta o estado termodinâmico do ar, variável ao longo da trajetória da gota. 


\section{Metodologia dos testes experimentais}

A temperatura da água do reservatório deveria, em princípio, ser a temperatura de ponto de orvalho do ar que sai de Vc2. Assim, sob condições ótimas, o ar sairia saturado, à temperatura do reservatório e, por posterior aquecimento, atingiria, também, a temperatura de bulbo seco desejada.

A operação da câmara climática depende, fortemente, da temperatura da água do reservatório e da troca de calor e massa entre o ar úmido que entrou na câmara e a água oriunda do spray e da superfície plana de água. Assim, exceto por ativação maior da resistência blindada ou do sistema de refrigeração, a câmara não depende de condições climáticas sob condições brasileiras.

Efetuaram-se, assim, no equipamento construído no Departamento de Engenharia Mecânica da UFMG, experimentos sob seis condições diferentes de temperatura da água do reservatório, na faixa entre 5,5 (temperatura mínima de funcionamento sem acúmulo de gelo) e $45^{\circ} \mathrm{C}$, com cinco repetições; as condições de teste foram de, aproximadamente, 5,5; 9,0;14,0;23,0;41,0 e 44,0 ${ }^{\circ} \mathrm{C}$; dada a flutuação das condições termodinâmicas do ar de entrada, houve, também, uma flutuação de até $0,5^{\circ} \mathrm{C}$ nesses valores. Por meio de válvula tipo borboleta, instalada na entrada da câmara, pôdese variar as velocidades do ar e, portanto, as vazões mássicas associadas; as velocidades locais médias ficaram entre 3,0 e 10,4 $\mathrm{m} \mathrm{s}^{-1}$, levando a valores calculados de vazões entre $31 \mathrm{e} 166 \mathrm{~g} \mathrm{~s}^{-1}$; esses valores de vazão dependem, também, dos dados termodinâmicos do ar de entrada (vazão mássica $=$ massa específica do ar úmido à entrada $\mathrm{x}$ velocidade $\mathrm{x}$ área do duto de entrada).

\section{RESULTADOS E DISCUSSÃO}

Tentou-se utilizar técnicas de ajuste estatístico multivariável para obterem os coeficientes empíricos das eqs.(2) e (4). O caráter não-linear das equações levou à formulação de metodologia de busca não-estatística para obter os coeficientes. Assim, por meio de várias simulações usando técnicas de busca disponíveis no IMSL (FORTRAN 95), obtiveram-se os coeficientes por comparação dos valores absolutos dos desvios relativos máximos e médios dos dados de simulação com os dados experimentais. A técnica de busca (método do gradiente) exigia a suposição de valores iniciais A e B [eqs.(2) e (4)]. Depois, refinaram-se os valores de A e B.

O ajuste dos modelos aos dados experimentais levou às seguintes expressões para os coeficientes de transporte de calor e massa, no interior da câmara climática, sobre a superfície da água:

a) Processos de umidificação do ar:

$$
\mathrm{h}=1,58 \mathrm{c}_{\mathrm{p}} \mathrm{URe}^{0,27} \operatorname{Pr}^{-2 / 3} \text { e } \mathrm{h}_{\mathrm{m}}=0,48 \rho \mathrm{URe}^{0,27} \mathrm{Sc}^{-2 / 3}
$$

b) Processos de desumidificação do ar:

$$
\mathrm{h}=0,8 \mathrm{c}_{\mathrm{p}} \mathrm{URe}^{0,27} \operatorname{Pr}^{-2 / 3} \text { e } \mathrm{h}_{\mathrm{m}}=0,58 \rho \mathrm{URe} \mathrm{R}^{0,27} \mathrm{Sc}^{-2 / 3}
$$

Os desvios relativos médios e máximos obtidos, ao se compararem os dados experimentais com os resultados das simulações, são apresentados na Tabela 4. Essa Tabela mostra que a modelagem proposta pode ser usada em projetos de câmaras complexas com o formato geométrico do condicionador apresentado, dentro dos desvios especificados. O uso de controladores de temperatura permitirá a operação adequada aos valores requeridos pelo projetista ou usuário.

TABELA 4. Valores absolutos dos desvios relativos médios e máximos entre os dados experimentais e simulados.

\begin{tabular}{llll}
\hline Desvios Relativos Médios & \multicolumn{3}{l}{ Desvios Relativos Máximos } \\
\hline Temperatura de bulbo seco & $2,06 \%$ & Temperatura de bulbo seco & $6,86 \%$ \\
Umidade absoluta & $2,00 \%$ & Umidade absoluta & $6,22 \%$ \\
Umidade relativa & $2,02 \%$ & Umidade relativa & $7,83 \%$ \\
\hline
\end{tabular}


Somente os dados experimentais das condições extremas foram utilizados para obterem os coeficientes A e B. Portanto, os dados de simulação, a serem mostrados, devem ser considerados como predições. Nas Figuras 3 a 8, apresentam-se exemplos dos dados experimentais de temperatura e umidade relativa do ar à saída da câmara climática e sua comparação com os resultados das simulações.

As temperaturas da água no reservatório nos testes citados foram 5,$5 ; 9 ; 14,3 ; 23,2 ; 41,5$ e $44{ }^{\circ} \mathrm{C}$. As condições de entrada, ou seja, as temperaturas de bulbo seco e umidades relativas foram, respectivamente, $23,2{ }^{\circ} \mathrm{C}$ e $54,5 \% ; 25,8{ }^{\circ} \mathrm{C}$ e $57,4 \% ; 24,2{ }^{\circ} \mathrm{C}$ e $70,1 \% ; 23,5^{\circ} \mathrm{C}$ e $74,0 \% ; 24,9{ }^{\circ} \mathrm{C}$ e $75,8 \%$ e $26,4^{\circ} \mathrm{C}$ e $63,8 \%$.

Os resultados mostrados nas figuras mostram que a modelagem apresentada reflete os fenômenos físicos complexos que ocorrem no interior da câmara climática. Deve-se notar que cada ponto experimental reflete um experimento quase que distinto do anterior, pois, ao se variar a vazão, as condições termodinâmicas internas à câmara mudam. Assim, em cada experimento, há a variação de pelo menos uma das variáveis: temperatura da água, temperatura de bulbo seco do ar, umidade relativa do ar e vazão de ar.

Deve-se notar, nas Figuras 3 a 8, que o interesse principal do projetista é manter a umidade relativa do ar de saída da câmara, no volume de controle 2, o mais próxima possível de $100 \%$, ou seja, da saturação. Assim, o ar úmido, na saída de Vc2 estará a um ponto de orvalho próximo ao da temperatura da água no reservatório. Um aquecimento posterior permitirá obter a temperatura de bulbo seco adequada.
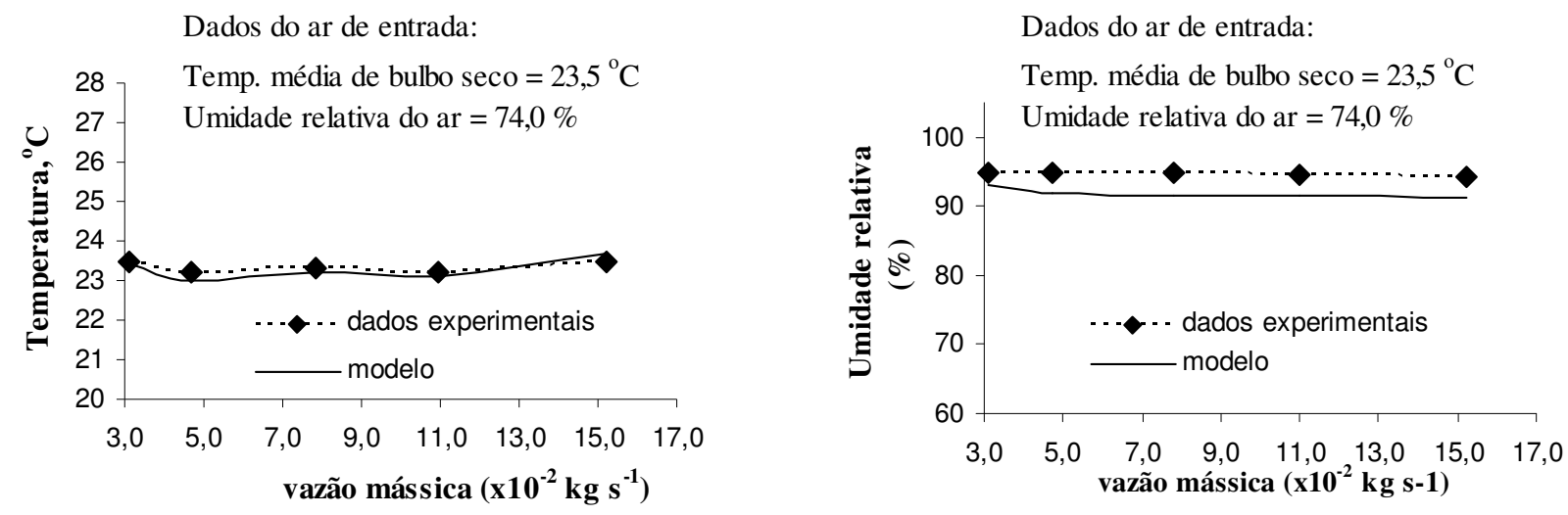

FIGURA 3. Temperatura e umidade relativa do ar à saída da câmara climática (temperatura da água $\left.=23,2{ }^{\circ} \mathrm{C}\right)$.
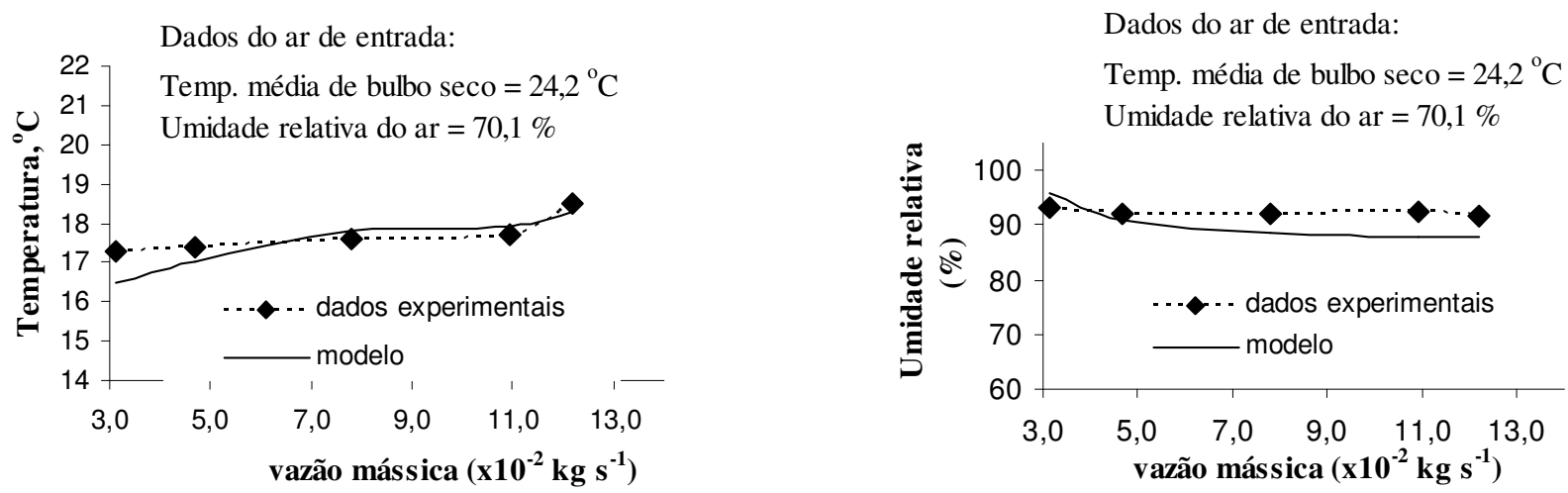

FIGURA 4. Temperatura e umidade relativa do ar à saída da câmara climática (temperatura da água $\left.=14,3{ }^{\circ} \mathrm{C}\right)$. 

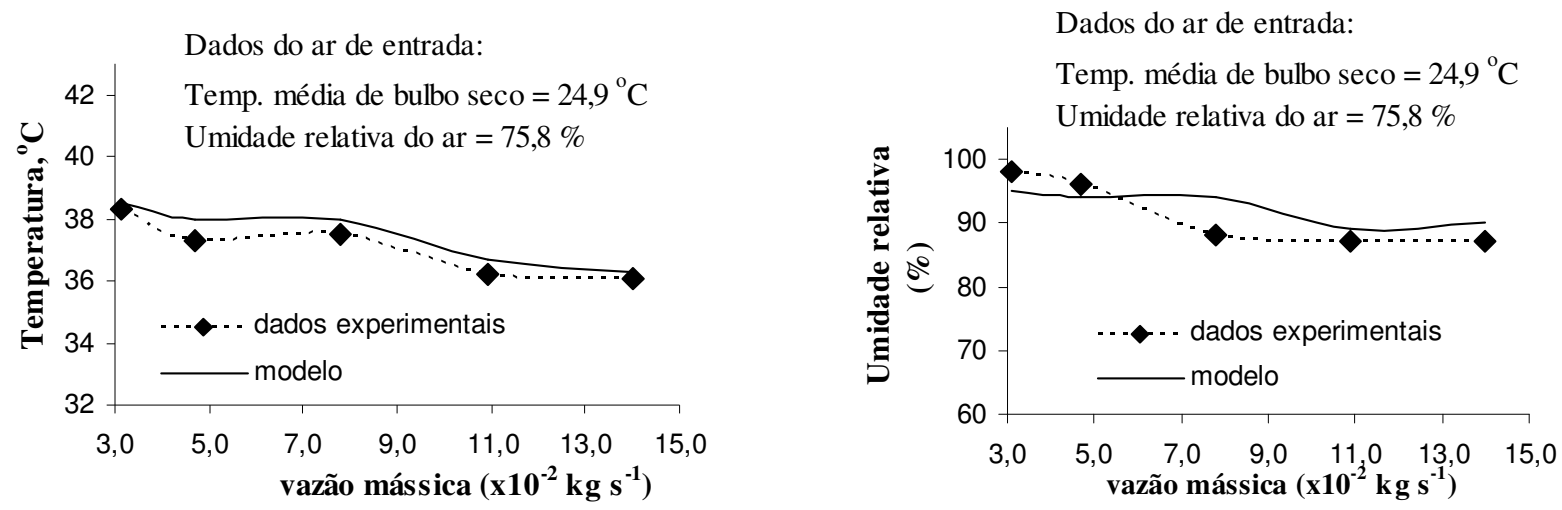

FIGURA 5. Temperatura e umidade relativa do ar à saída da câmara climática (temperatura da água $\left.=41,5^{\circ} \mathrm{C}\right)$.
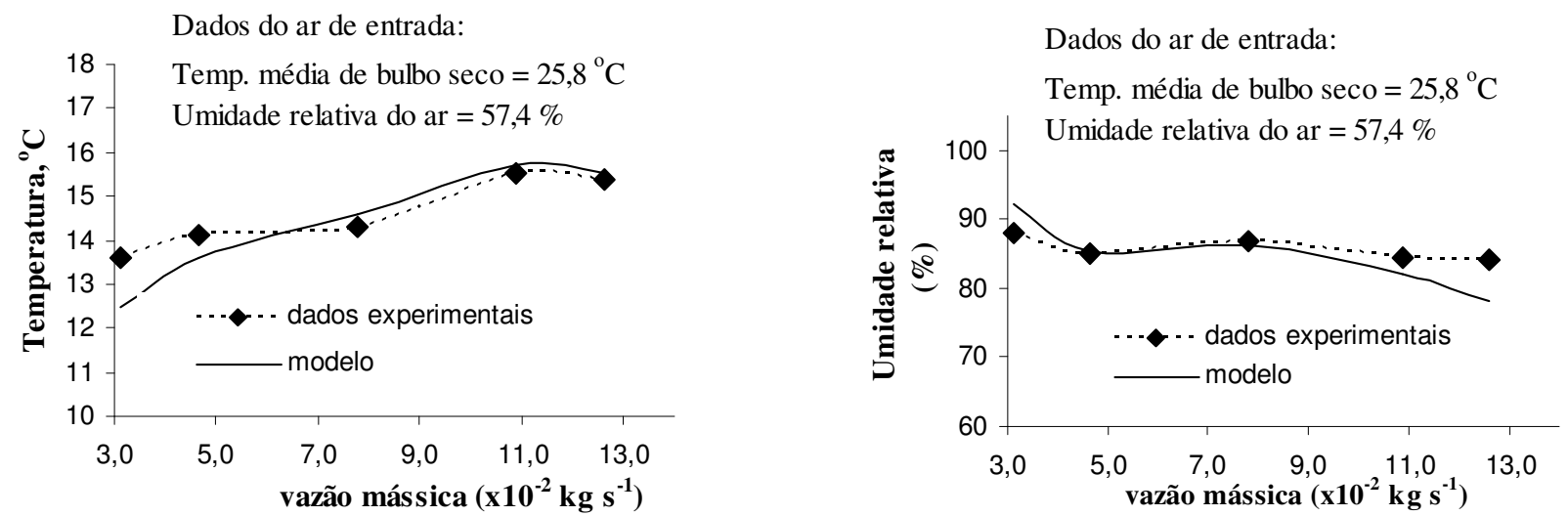

FIGURA 6. Temperatura e umidade relativa do ar à saída da câmara climática (temperatura da água $=9{ }^{\circ} \mathrm{C}$ ).
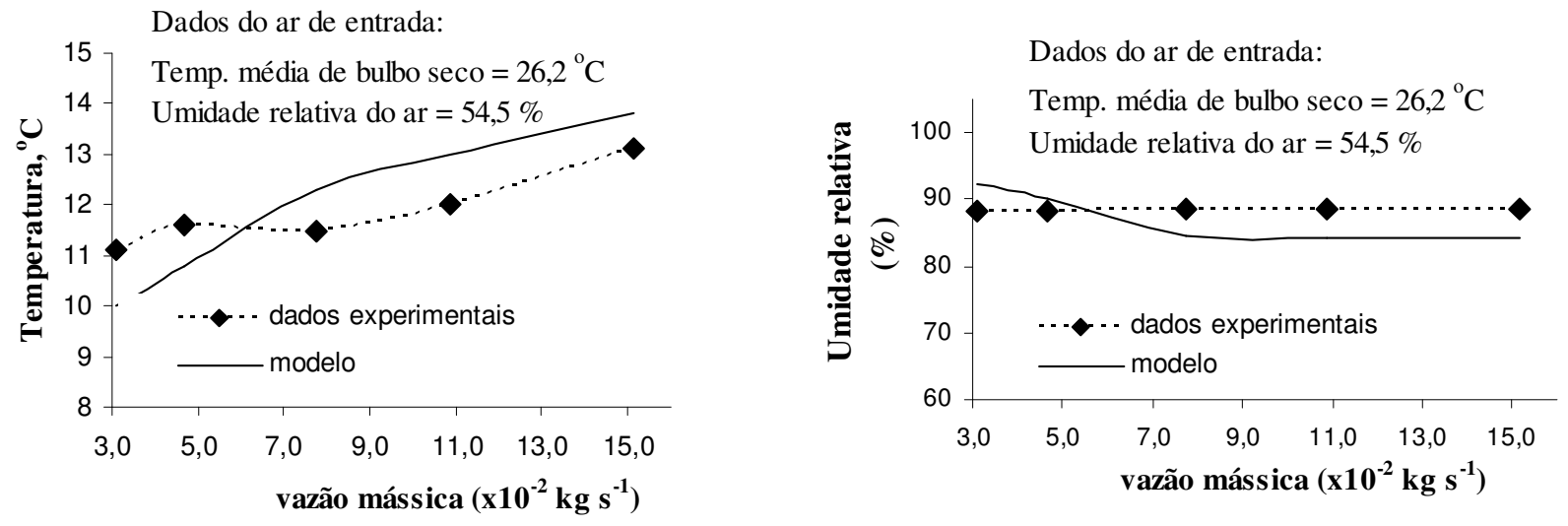

FIGURA 7. Temperatura e umidade relativa do ar à saída da câmara climática (temperatura da água $=5,5^{\circ} \mathrm{C}$ ). 

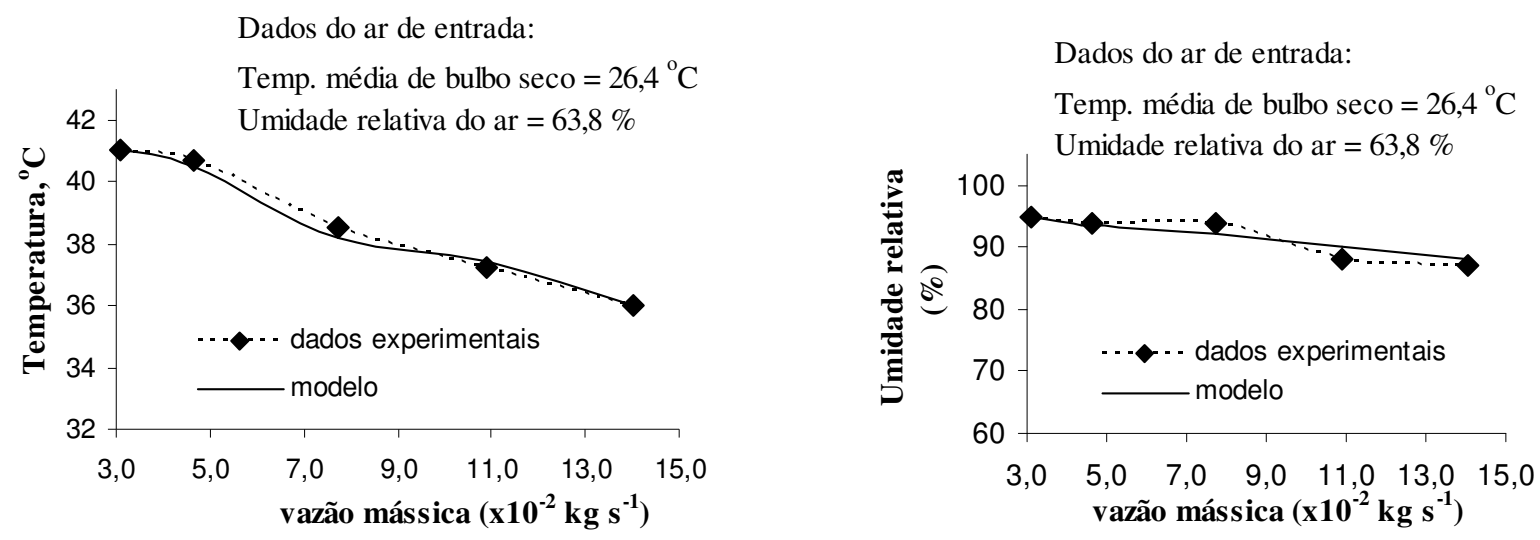

FIGURA 8. Temperatura e umidade relativa do ar à saída da câmara climática (temperatura da água $=44,5^{\circ} \mathrm{C}$ ).

\section{CONCLUSÕES}

A modelagem desenvolvida e os testes experimentais permitiram obter os coeficientes de transferência de calor e massa para uma corrente de ar úmido sobre uma superfície plana de água e o ajuste de expressões para as condições reinantes no interior da câmara condicionadora. Assim, concluiu-se que os coeficientes empíricos ajustados dependem tanto do formato geométrico do recipiente quanto das ondulações causadas pela sucção feita pela bomba d'água do reservatório.

O ajuste dos modelos globais de simulação aos dados experimentais, de maneira a minimizar desvios absolutos médios e máximos dos valores de temperatura e umidade relativa de saída em função da vazão mássica, levou a desvios relativos máximos de 6,86\%; 6,22\% e 7,83\% nos valores respectivos de temperatura de bulbo seco, umidade absoluta e umidade relativa.

Uma vez que o modelo global proposto teve por base equações definidas em termos de números adimensionais, pode-se supor que condicionadores de ar com geometria semelhante e vazões mássicas variáveis possam ser projetados utilizando a metodologia proposta.

\section{REFERÊNCIAS}

BIRD, R.B.; STEWART, W.E.; LIGHTFOOT, E.N. Transport Phenomena. $2^{\text {nd }}$ ed. New York: Wiley, 2001. $912 \mathrm{p}$.

BONACINA, C.S.; DEL GIUDICE, S.; COMINI, G. Dropwise evaporation. Journal of Heat Transfer, Cambridge, v.101, n.3, p.401-41, 1979.

FERREIRA, W.R.; FORTES, M.; BRETON, J.L. A numerical and experimental study of natural convective heat and mass transfer from a vertical porous plate. I. Numerical analysis. Drying Technology, Quebec, v.11, n.7, p.1657-84, 1993a.

FERREIRA, W.R.; FORTES, M.; BRETON, J.L. A numerical and experimental study of natural convective heat and mass transfer from a vertical porous plate. II. Holografic interferometry experimental analysis. Drying Technology, Quebec, v.11, n.7, p.1685-1712, 1993b.

FIRST, N.L.; PARRISH, J.J. In vitro fertilization of ruminants. Journal of Reproduction Fertility, Cambridge, v.34, p.151-65, 1987.

HOLMAN, J. P. Heat transfer. $9^{\text {th }}$ ed. Boston: Mcgraw-Hill, 2002. 665 p.

KEEY, R.B. Drying principles and practices. $4^{\text {th }}$ ed. Oxford: Pergamon, 1972. 358 p.

NASR, G.G.; YULE, A.J.; BENDIG, L. Industrial sprays and atomization: design, analysis and applications. New York: Springer Verlag, 2002. 500 p. 
SINGH, R.P.; HELDMAN, D.R. Fundamentals of food process engineering. New York: Academic Press, 2001.630 p.

STOECKER, W.F.; JONES, J. Refrigeração e ar-condicionado. São Paulo: McGraw-Hill, 1985. $481 \mathrm{p}$.

TREYBAL, R.E. Mass-transfer operations. $3^{\text {rd }}$ ed. Auckland: McGraw-Hill, 1981. 784 p.

YULE, A.J.; SHARIEF, R.A.; JEONG, J.R.; NASR, G.G.; JAMES, D.D. The performance characteristics of solid-cone-spray pressure-swirl atomizers. Journal of the International Institute for Liquid Atomization and Spray Systems, Pittsburgh, v.10, n.6, p.21-41, 2002. 